\title{
Soft mold and gasbag pressure mechanism for patterning submicron patterns onto a large concave substrate
}

\author{
Fang-Sung Cheng and Sen-Yeu Yang ${ }^{a)}$ \\ Grace Laboratory for Polymer Processing, Department of Mechanical Engineering, National Taiwan \\ University, Taipei, Taiwan 106, Republic of China \\ Shih-Chih Nian \\ Department of Mechanical Engineering, Dahan Institute of Technology, Taipei, Taiwan 106, \\ Republic of China \\ Lon A. Wang \\ Department of Electrical Engineering, National Taiwan University, Taipei, Taiwan 106, Republic of China \\ and Graduate Institute of Electro-Optical Engineering, National Taiwan University, Taipei, Taiwan \\ 106, Republic of China
}

(Received 11 January 2006; accepted 10 May 2006; published 28 June 2006)

\begin{abstract}
A gasbag pressure (GBP) mechanism has been developed for patterning submicron patterns onto large concave substrate. The GBP mechanism consists of a pressure gasbag and a vacuum chamber system. It provides gradual contact, uniform pressure, and intact contact for imprinting patterns in the soft mold onto a concave substrate. The patterns on the soft mold can be successfully replicated over an entire photoresist-coated concave substrate. The accuracy of replication has been experimentally evaluated. (ㅇ 2006 American Vacuum Society. [DOI: 10.1116/1.2209991]
\end{abstract}

\section{INTRODUCTION}

Replication of submicron patterns onto curved surfaces has been a recent matter of growing interest, especially for the fabrication of optics and sensor components. ${ }^{1,2}$ For example, the Rowland circle spectrograph has gratings on a concave surface to separate incipient light from a slit source. ${ }^{2}$ These can be made by focused laser irradiation of solids and electron beam ion traps. However, it is time consuming and not cost effective. Besides, curved surfaces are difficult to pattern using conventional nanoimprint lithography ${ }^{3}$ (NIL) or ultraviolet-based NIL. ${ }^{4}$ The reason is that conventional silicon and quartz molds cannot easily retain conformal contact with the substrate when the substrate is nonplanar. In modern technology, submicron patterning of 1 in. diameter curved surface with a $46 \mathrm{~mm}$ radius of curvature has been demonstrated with step and flash imprint lithography (SFIL) using templates patterned by ion beam proximity (IBP) printing. ${ }^{5}$ The features on the mask will only be in focus in a small area of the exposed region. This method is extremely time consuming for fabrication. Whitesides and co-workers ${ }^{6,7}$ used a poly(dimethylsiloxane) (PDMS) mold as an elastomeric phase mask to pattern nanostructures on hemispherical and cylindrical surfaces. The PDMS mold, combined with a vacuum system, can provide conformal contact and pressure for patterning between the flexible mold and convex substrate. However, if the surface is large or the shape of the surface is concave, the residual gas may still be trapped in the substrate even after vacuuming, since the maximum pressure is only $1 \mathrm{~atm}$. Choi and

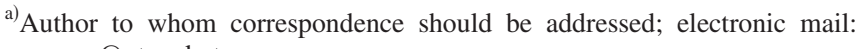
syyang@ntu.edu.tw
Park $^{8}$ used a similar PDMS mold to imprint microfeatures onto cylinders; the pressure was applied by hand to keep substrates and soft mold in contact.

To enhance close contact and uniform pressure when imprinting large surfaces, Chang et al. ${ }^{9}$ used gas pressure to compress the mold/substrate stack. Microstructures on 2 in. convex surface and 12 in. silicon wafer have been successfully replicated by using the gas-pressuring mechanism. However, it is still difficult to pattern on a concave surface due to air entrapment when the edge is enclosed by a sealing film.

The objective of this study is to construct a simple and cost-effective system for replicating microfeatures onto curved, especially concave, surfaces. In addition to the use of the soft mold and gas-assisted pressuring scheme, gasbag arrangement is proposed and implemented for imprinting microfeatures on the soft mold onto concave surfaces. Gasbag can ensure air venting since pressuring starts from the center and gradually extends outward. In this study, the feature size between the replicated and original microfeatures will be compared, and its association with the deformation of the soft mold will also be analyzed.

\section{GASBAG PRESSURING PROCESS}

Figure 1 shows the mechanism of the gasbag pressuring (GBP) process. It consists of a vacuum chamber system and a gasbag pressure system. The vacuum chamber system is used to reduce air before imprinting. The gasbag system provides gradual contact between the mold and curved substrate, from the center outwards, and uniform pressure.

The imprinting process can be divided into three steps: Firstly, a concave glass is dip-coated with photoresist, and a PDMS mold with microfeatures is placed above it to form a stack, which is enclosed in the chamber. Secondly, after the 


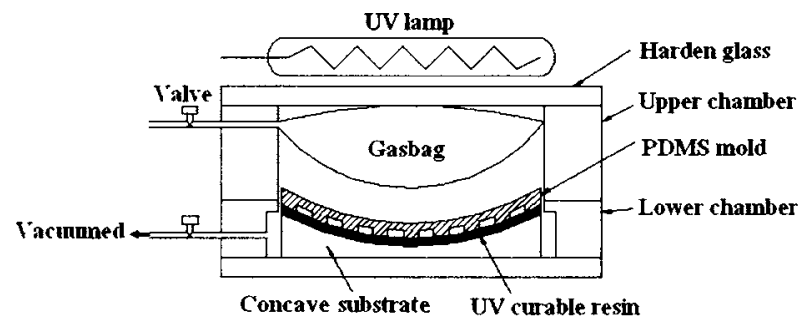

(a)

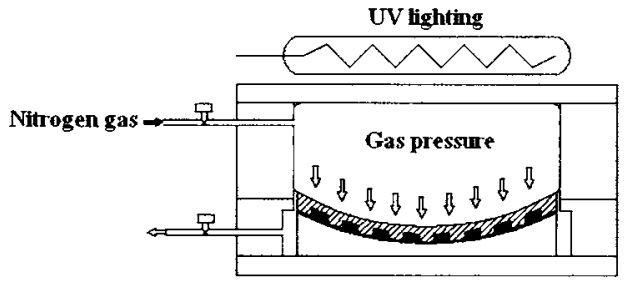

(b)

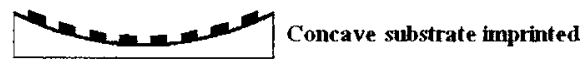

(c)

FIG. 1. Schematic of the mechanism and process flow of gasbag pressure (GBP) for the fabrication of submicron patterns on the concave substrate.

chamber is closed and vacuumed, nitrogen is introduced into the gasbag to press the stack. Then, the photoresist is exposed to UV light to be cured. Finally, the vacuum chamber and gasbag are released to retrieve the imprinted substrate. This soft mold/gasbag pressuring scheme is to be used for the imprinting of submicron patterns on a concave surface.

\section{EXPERIMENTAL SETUP}

\section{A. Preparation of PDMS molds}

The patterns of the 4 in. diameter silicon master were first replicated onto polycarbonate (PC) films using a gaspressurized hot embossing process ${ }^{10}$ as shown in Fig. 2(a). Then the poly(dimethylsiloxane) (PDMS, Sylgard ${ }^{\mathrm{TM}}$ 184, Dow Corning) resin is coated on the PC films as shown in Fig. 2(b). The thin planar PDMS mold is cured at room temperature for one day. A series of pattern squares (3 $\times 3 \mathrm{~mm}^{2}$ ) with various patterns are formed. The photographs of silicon mold, PC film, and PDMS mold are shown in Fig. 2(c). Chang et al. ${ }^{9}$ proved that the dimensions and shapes of the replicated patterns on the PDMS mold are very close to those in the original silicon mold. The use of PDMS molds has another advantage. It can eliminate the problem of sticking during detachment, since PDMS has low surface energy at the polymer interface. In this study, the replica dot microstructures with the diameter of $600 \mathrm{~nm}$ were used for analytical comparison between the replicated and original microfeatures.

\section{B. Preparation of imprint substrates and photoresist}

The large concave substrate used is $70 \mathrm{~mm}$ diameter in baseline and $57.5 \mathrm{~mm}$ radius in curvature. The material is glass. An UV curable epoxy resin (UR40, S-Light Optoelectronics, Ltd.) is dip-coated onto the concave surface. The

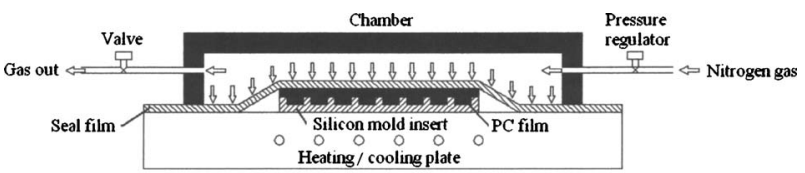

(a)

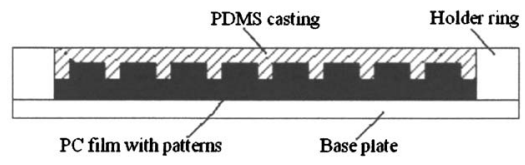

(b)
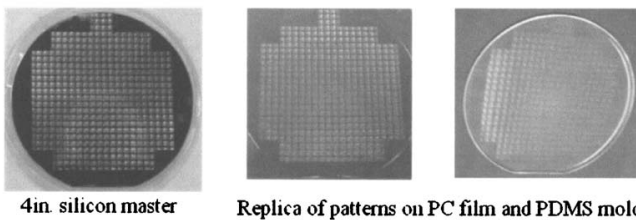

Replica of patterns on PC film and PDMS mold

(c)

FIG. 2. (a) Schematic drawing of a gas-pressurized hot embossing process. (b) Schematic illustration of the casting of poly(dimethylsiloxane) (PDMS) on the PC films. (c) Photograph of the silicon mold, PC film, and PDMS mold.

resin is a colorless, clear liquid with a viscosity of $500 \mathrm{cPs}$ at $25^{\circ} \mathrm{C}$. The exposure intensity of the resist is $300 \mathrm{~mJ} / \mathrm{cm}^{2}$. The resist can be used at room temperature and with low pressure.

\section{Imprinting conditions}

The vacuum pressure is $-0.5 \mathrm{~kg} / \mathrm{cm}^{2}$ and the gasbag pressure is $1 \mathrm{~kg} / \mathrm{cm}^{2}$. The operation temperature is $25^{\circ} \mathrm{C}$ in all of the experiments. The substrate is exposed to UV irradiation for 6 min through the PDMS mold and transparent gasbag. The power of UV lamp (UL, LISTED 977C) is $800 \mu \mathrm{W} / \mathrm{cm}^{2}$ and the used wavelength range from 365 to $410 \mathrm{~nm}$.

\section{RESULTS}

\section{A. Comparison of imprinting of a large concave substrate with and without gasbag}

Figure 3(a) shows a photograph of a concave surface imprinted without the gasbag pressure mechanism. It was imprinted directly with gas pressure applied on the PDMS

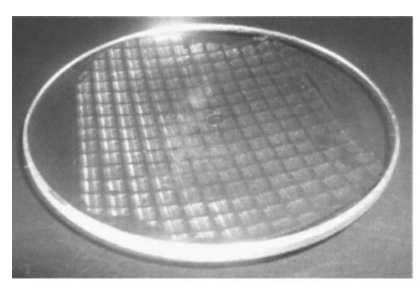

(a)

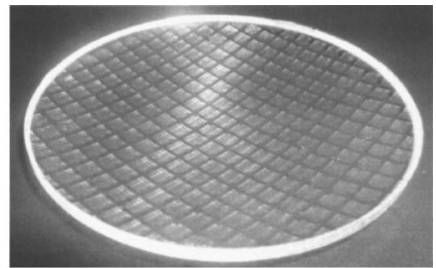

(b)
FIG. 3. Photograph of imprinted patterns on a concave substrate $(70 \mathrm{~mm}$ diameter in baseline with $57.5 \mathrm{~mm}$ radius of curvature) (a) without and (b) with gasbag pressure mechanism. 


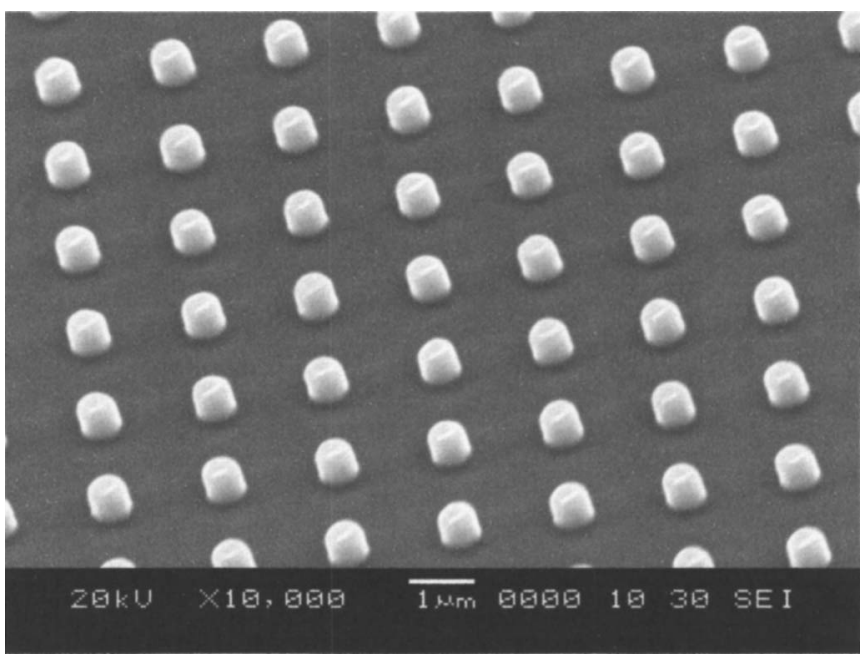

FIG. 4. SEM images of a stereograph of dots array with $600 \mathrm{~nm}$ in standard diameter. The shapes of patterns on the concave surface are fairly straight and vertical.

mold/glass substrate concave stack. Since residual air is trapped in the concave surface, air bubbles were found in the center zone. By imprinting with a gasbag pressure mechanism, the vacuum chamber system is used to reduce air before imprinting, and the gasbag system is used to provide gradual contact between the mold and curved substrate, from the center outwards, and final uniform pressure. The whole imprinted area is free of air bubbles or defects as shown in Fig 3(b). The PDMS mold and gasbag pressure mechanism can achieve the close contact and uniform pressing without air trapping. Figure 4 shows the scanning electron microscope (SEM) photograph of a series of replicated dots. The standard diameter is $600 \mathrm{~nm}$ and the standard height is $350 \mathrm{~nm}$. The SEM images of the replication show the fine shapes of patterns on the concave surface. These results indicate that the GBP mechanism with PDMS mold can lead to a uniform imprinting pressure with conformal contact on the concave substrates.

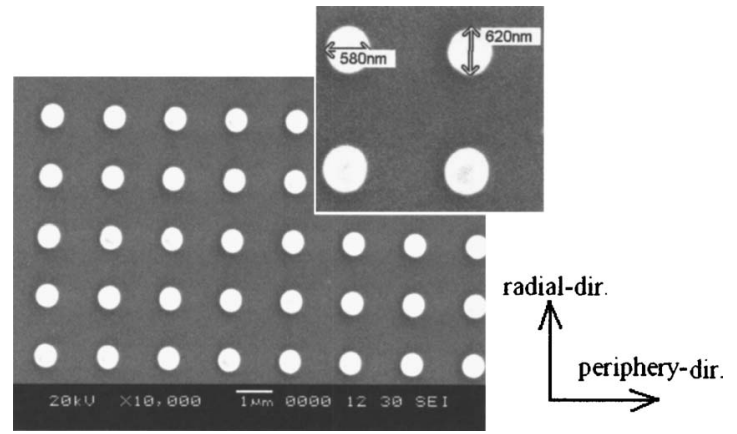

FIG. 5. SEM image of a plane of dots array with $600 \mathrm{~nm}$ in standard diameter. On the peripheral direction of the concave substrate, the diameter is $580 \mathrm{~nm}$; on the radial direction of the concave substrate, the diameter is $620 \mathrm{~nm}$.

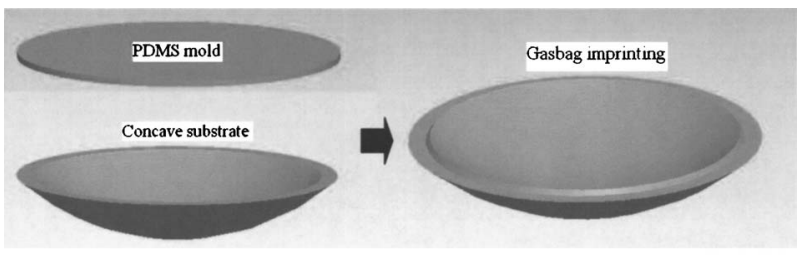

(a)
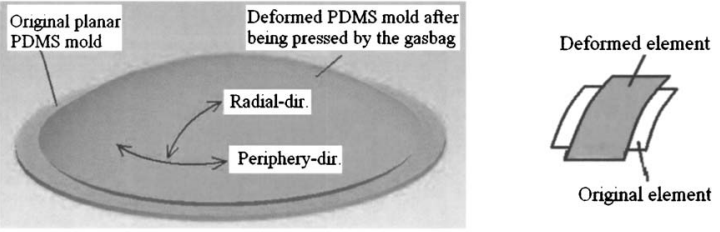

(b)

FIG. 6. (a) Schematic drawing of the flexible PDMS mold, which is capable of adapting its shape to the whole concave surface. (b) Schematic illustration of the flexible PDMS mold pressed against the concave surface; the strain in the peripheral direction is -0.035 and is 0.035 in the radial direction.

\section{B. Observation on the dimensions of imprinted dots on the concave surface}

Detailed measurements show that there is dimensional distortion in the replications on large concave surfaces. Figure 5 shows that the diameter is $580 \mathrm{~nm}$ in the peripheral direction; the diameter is $620 \mathrm{~nm}$ in the radial direction. It is observed that the flexible PDMS mold is capable of adjusting its shape to the whole concave surface as shown in Fig. 6(a), resulting in geometrical distortion as shown in Fig. 6(b). When the flexible PDMS mold is pressed against the concave surface, the strain in the peripheral direction is -0.035 , and it is 0.035 in the radial direction.

\section{CONCLUSIONS}

Submicron features are patterned onto a concave substrate by a combination of soft lithography with GBP mechanism and UV-NIL. The replication shows that it can avoid air trapping. Over the whole concave surface, there are no air bubbles. The shapes of replication patterns are fairly straight, vertical, and clear. However, the measured dimensions show different distortions in two directions due to the nonplanar deformation of a flexible material on concave surfaces. The dimension is enlarged in the radial direction, but it is reduced in the peripheral direction. These imply that the use of this method has its limitation if high precision is demanded.

\section{ACKNOWLEDGMENT}

This work was supported by the National Science Council under Contract No. NSC 94-2218-E-002-037. Special thanks to S-Light Optoelectronics, Ltd. for the supply of commercial UV curable resist (UR40). The authors also thank coworkers in Professor Wang's laboratory for carrying out the dimensional measurements and co-workers in the Grace Laboratory for providing experimental assistance. 
${ }^{1}$ H.-C. Jin, J. R. Abelson, M. K. Erhardt, and R. G. Nuzzo, J. Vac. Sci. Technol. B 22, 2548 (2004).

${ }^{2}$ R. Barnsley, N. J. Peacock, J. Dunn, I. M. Melnick, I. H. Coffey, J. A. Rainnie, M. R. Tarbutt, and N. Nelms, Rev. Sci. Instrum. 74, 2388 (2003).

${ }^{3}$ S. Y. Chou, P. R. Krauss, and P. J. Renstrom, Appl. Phys. Lett. 67, 3114 (1995).

${ }^{4}$ U. Plachetka et al., Microelectron. Eng. 73\&74, 167 (2004).

${ }^{5}$ P. Ruchhoeft et al., J. Vac. Sci. Technol. B 17, 2965 (1999).
${ }^{6}$ A. Rogers, K. E. Paul, R. J. Jackman, and G. M. Whitesides, Appl. Phys. Lett. 70, 2658 (1997).

${ }^{7}$ K. E. Paul, M. Prentiss, and G. M. Whitesides, Adv. Funct. Mater. 13, 259 (2003).

${ }^{8}$ W. M. Choi and O. O. Park, Nanotechnology 15, 1767 (2004).

${ }^{9}$ J. H. Chang, F.-S. Cheng, C.-C. Chao, Y.-C. Weng, S. Y. Yang, and L. A. Wang, J. Vac. Sci. Technol. A 23, 1687 (2005).

${ }^{10}$ J. H. Chang and S. Y. Yang, Microsyst. Technol. 10, 76 (2003). 